\title{
At the crossroads: molecular ecology and wildlife
}

\section{conservation}

\author{
Uma Ramakrishnan
}

Biodiversity surrounds us. We are enthralled by its beauty and variety. But how has this diversity come to be? How do we quantify it? What threatens it? How do humans impact biodiversity? Can we understand more about and help suggest interventions that will allow species to survive?

Genetic material is one thread that weaves all these questions together. Using genetic sequence and variations we find therein, we can answer all these questions. The field that uses genetic tools to answer these questions is called molecular ecology, and in this special issue of the IISc journal, we bring together several systematic reviews in this field.

Biodiversity is quantified in units of species, and species delineation can be conducted using integrative taxonomy, which includes genetic data. Joshi and Agarwal review the integrative taxonomy efforts for vertebrates and invertebrates, with a focus on South Asia, and suggest constructive approaches to deal with the large data gaps we face in India. Fundamental to describing diversity is a better understanding of processes that drive its creation. Biswas and Karanth review 2 decades of research on species in the Western Ghats to investigate whether biogeographic barriers, such as gaps in mountain ranges, result in speciation. Finally, speciation, and the subsequent generation of diversity, is contingent on the lack of gene flow. In scenarios and species where gene flow is difficult, but possible, what does gene flow depend on? Chattopadhyay and Garg review gene flow in vertebrates capable of flight, and highlight specifically the gaps in knowledge in biodiversity rich areas like tropical Asia.

Humans have significantly impacted the earth. Several of the articles in this special issue deal with studying the fallouts of how humans are impacting our planet and species. Globally, humans have added greenhouse gases to the atmosphere, and this has resulted in increasing temperatures and global change. Such changes are felt most strongly by species that live in mountains. Dahal et al. review how species in the
Himalaya will respond to climatic change, and how genetic data from across species' genomes can be used to investigate how species have responded to changes in climate. Apart from global effects, human activities have also resulted in significant habitat alteration and degradation. As an immediate effect, such habitat fragmentation can result in the loss of connectivity for wildlife species. Thatte et al. present a comprehensive review of connectivity studies in South Asia, and show that several studies lack behavioral data and/or movement data. Human effects can be compounded in cases of complex evolutionary processes like disease. Ishtiaq reviews avian malaria studies in the tropics and shows that both habitat fragmentation and climate change can impact disease dynamics. On the other hand, Sreedharan and Vasudevan review literature on chytridiomycosis, a fungal disease in amphibians that appears less prevalent in Asia. They discuss possible reasons and knowledge gaps, and how molecular tools can aid to bridge these gaps.

Molecular tools can help us understand more about the biology of specific endangered species. Athira and Vidya review literature on elephant social groups, and how molecular tools have contributed to our understanding of their social systems, and highlight how the social systems of these intelligent, social animals might be affected by humans. Weckworth reviews our gaps in understanding of another charismatic and endangered species, the snow leopard.

Through these reviews, several themes emerge, that gaps in knowledge tend to be concentrated where knowledge is most needed, biodiverse continents like Asia! Khan and Tyagi review the possible reasons for the lack of genome-wide studies in the biodiverse tropics, and suggest a way forward to enable conservation genomics in poor resource settings.

Overall, the papers presented here address a rich array of biodiversity. In putting together this issue, I have been biased, and focused on Indian/Asian biodiversity. It has been a pleasure
${ }^{1}$ National Centre for Biological Sciences, TIFR, Bangalore, India. *uramakri@ncbs.res.in 
to work with and hear from the many early career researchers who are sure to fill these knowledge gaps in the years to come. Given the collective expertise showcased in this issue, the future of molecular ecology and wildlife conservation is bright!

I thank the IISc journal team, especially Ms. M. V. Leelakshi and Ms. Kavitha Harish, for their tremendous patience. Thanks to the editorial team including Prof. Rashna Bhandari and editorin-chief, Prof. G. K. Ananthasuresh for the opportunity to edit this special issue. The final revisions and proofing took place during the second wave of COVID-19 in India, and I thank all the authors and journal staff for their resilience and patience in the face of a pandemic that touched almost every one of us personally, with loss or ill-health. That said, the pandemic convinces us that we must regain a balance with nature. As scientists, we must continue our exploration of nature, so we can better understand biodiversity, and how we can sustain it on our planet. Because we know now better than ever, that our future too, depends on such understanding.

\section{Publisher's Note}

Springer Nature remains neutral with regard to jurisdictional claims in published maps and institutional affiliations.

Published online: 5 July 2021 